\title{
Ai-je mérité mon tee-shirt?
}

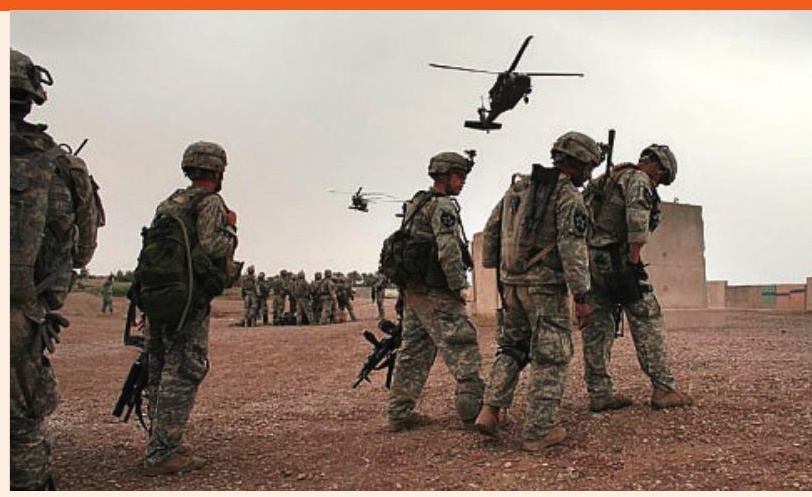

Irak-Bienne-retour ... sur un tapis.

Françoise Verrey Bass

Correspondance:

Françoise Verrey Bass

Plänkestrasse 12

CH-2502 Bienne

fraverrey@gmx.ch
Qu'est ce que Bienne, la petite ville au pied du Jura, peut bien avoir en commun avec la guerre en Irak?

Bienne, au début du siècle passé une gentille petite ville, bien calme, romantique, ses bras de la Suze, son petit fleuve personnel garni de belles maisons bourgeoises entourées de jardins bucoliques. Une petite ville horlogère, assidue, où le chemin de fer venait d'installer sa gare au centre ville d'où partaient les diligences pour mener les voyageurs acheteurs de montres dans les autres localités horlogères du Jura, Saint-Imier, La Chaux-de-Fonds, Le Locle et autres. La population augmentait gentiment, régulièrement, la ville prospérait. Dès le milieu du siècle elle se sentit des ailes, des envies de grand monopole. Longtemps avant que l'on ne parle d'Union européenne elle s'intitula ville de l'avenir, ville de l'Europe. Mais pour être à la hauteur de ses ambitions il faut se faire connaître. Comment! Macolin était en train de se faire, le centre de sport de Suisse, centre de formation, aujourd'hui devenue même université du sport, qui entraîna d'autres institutions devenues très connues comme par exemple l'école Swisstennis, qui hébergea notre héros national, Roger Federer, l'incontournable numéro 1 mondial de tennis.

Mais nous n'en sommes pas encore là. Revenons au milieu du siècle passé, aux années cinquante. Que faire pour parer la ville et la région d'un attrait exceptionnel, quelque chose qui devrait se répéter régulièrement pour attirer tout aussi régulièrement les gens à Bienne?

L'idée de génie jaillit: les $100 \mathrm{Km}$ de Bienne! Quelques années plus tard la chose était en place, un parcours de $100 \mathrm{~km}$ délimité autour de la ville, surtout allant vers le sud, les bourgades de Lyss et d'Aarberg, à l'est jusqu'à Büren an der Aare. Un parcours plein de suspense. Et le summum de la course: départ à $22 \mathrm{~h}$, le $2^{2 \mathrm{eme}}$ ou $3^{\text {ème }}$ week-end de juin, le vendredi soir. Ainsi, celui qui le désire peut être de retour au travail le lundi matin ... Course de nuit pour ne pas avoir trop de problèmes de coup de chaleur et autres pépins graves. Ceci est tout à fait juste pour les premiers, mais suivant le temps qu'il fait, les problèmes surgissent de jour, surtout sur la fameuse piste de Ho Chi Minh, très exposée au soleil ou complètement dans la gadoue quand inondée. Elle a disparu entre temps, cédant à l'autoroute de Soleure. Il n'y a pas que le tracé de la course qui a changé, s'est modernisé depuis cette première édition en 1959 et qui depuis se répète chaque année, cette année pour la $46^{\text {ème }}$ fois. La course est devenue mondialement connue, des équipes viennent et reviennent chaque année. Les femmes peuvent aussi courir depuis la moitié des années soixante. Le temps passant la course s'est restructurée, enjolivée, est devenue une fête populaire: il y a la course marathon de $42 \mathrm{~km}$, le parcours spécial pour les inline skaters, la course fragmentée, le parcours enfants. Les fonctionnaires sont aussi fidèles que les coureurs, reviennent chaque année pour organiser bénévolement ravitaillement, haltes de massages en cours de route et j'en passe. Il y a les coureurs en solo, les groupes, il $\mathrm{y}$ a les jeunes qui courent pour la première fois, les vétérans qui ont 20 courses et plus dans les jambes et se retrouvent chaque année avec un plaisir évident pour ressortir les anecdotes des courses passées. Le coureur le plus âgé avec 78 ans a fait la course en 16 heures. Plusieurs années de suite il y a eu un messager boiteux avec une jambe de bois, qui terminait la course!

Il y a les patrouilles militaires suisses et étrangères qui courent le jour avant, un trajet un peu plus sophistiqué. Bref, il y a de tout, vraiment de tout. Par contre les coureurs qui sont là depuis les premières années deviennent rares.

Il y a les accompagnants en vélo pour ravitailler leur coureur, l'encourager, le stimuler. D'autres véhicules ne sont pas autorisés exception faite de la voiture balais traînant derrière le dernier coureur qui n'abandonne pas, la lanterne rouge. Celui qui abandonne est ramené à Bienne. Pendant toute la course il y a des spectateurs tout au long du parcours pour encourager de vive voix les coureurs, leur donner de l'eau, du sucre de raisin et autres bâtons énergétiques. Toute la population des régions parcourues est sur pied comme il se doit.

Il y a les records de temps, le temps le plus court, mais aussi le temps le plus long. Le premier coureur de cette année était deuxième l'année passée avec un meilleur temps que cette année. Sur $100 \mathrm{~km}$ tout devient possible. Les meilleurs temps se situent autour des 7 heures 30. Il y a des records plus importants, le même gagnant 5 années de suite, ce qui est arrivé deux fois. Chez les femmes c'est plus rare de gagner deux années de suite.

Depuis quelques années chaque participant reçoit un tee-shirt avec l'inscription $100 \mathrm{Km}$ de Bienne et l'année correspondante, à condition qu'il termine la course. 
Cette année effervescence maximale à quelques heures du départ quand le téléphone sonne une fois de plus. Mais cette fois-ci il fallut d'abord trouver un interlocuteur parlant l'anglais. A l'autre bout du fil un colonel anglais stationné quelque part en Irak et demandant pour un de ses hommes l'autorisation de participer à la course - en Irak, au campement, sur un tapis - non pas volant, mais roulant! Stupeur et interrogation. Et puis, pourquoi pas? On verra bien.

A l'heure du départ, la communication avec l'officier britannique est rétablie. Ainsi le collègue coureur en Irak peut prendre son départ à l'instant même où le peloton à Bienne se met en route. L'histoire ne dit pas comment le soldat britannique passa la nuit, mais au matin, pas même tard, nouveau coup de téléphone du colonel britannique: «Hello, mon soldat a bouclé ses $100 \mathrm{~km}$. Il va bien. Il demande, si malgré les conditions exceptionnelles de sa course il pourra avoir son tee-shirt de participation, thanks. Il reviendra courir l'année prochaine comme depuis 6 ans, s'il est encore en vie, inch Allah!»

\section{Buchbesprechung}

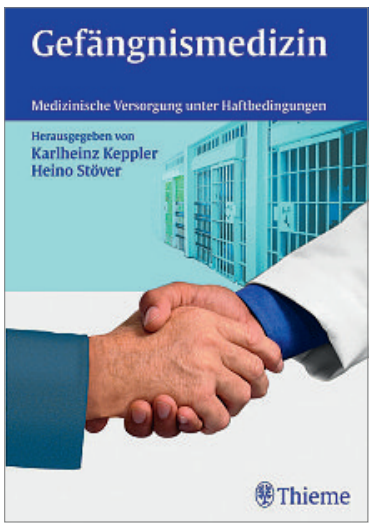

1 Bertrand D, Niveau G. Médecine, santé et prison. Editions Médecine et Hygiène: 2006; 484 Seiten. Fr. 82.- /€ 50,75. ISBN 2-88049-230-0.

\section{Gefängnismedizin}

Karlheinz Keppler, Heino Stöver (Hg.)

\section{Gefängnismedizin - Medizinische Versorgung} unter Haftbedingungen

Georg Thieme Verlag. Stuttgart: 2009. 344 Seiten. Fr. 149.-/€ 89,95. ISBN 978-3-13-147731-6.

\section{Arbeit in der totalen Institution}

Die beiden Herausgeber sind den vom Thema Betroffenen auch über die Landesgrenzen hinaus als Verfasser und Organisatoren fachspezifischer Tagungen her bekannt. Karlheinz Keppler ist Professor im Fachbereich Soziale Arbeit und Gesundheit an der Fachhochschule Frankfurt a. M., Heino Stöver Medizinaldirektor in der Justizvollzugsanstalt für Frauen in Berlin. Im Vorwort geben die Herausgeber ihren Bedenken wegen der möglichen Entwicklung einer bundeslandspezifischen Ausprägung der Gefängnismedizin in Deutschland Ausdruck als Folge der auf die einzelnen Länder übertragenen Zuständigkeit für den Justizvollzug. Verglichen mit der gegenwärtigen Organisation und dem Status quo in der Schweiz - oder mit Blick auf die zentrale Organisation z.B. in Frankreich - ist diese Befürchtung nicht unbegründet.

Das vorliegende Buch entstand, wie die Herausgeber schreiben, «im Sinne der multiprofessionellen und interdisziplinären Kooperation, welche die Gesundheitsdienstleistung der Gefangenen darstellt», unter Mitarbeit von über dreissig Fachleuten aus Praxis, Forschung und Lehre der somatischen und psychiatrisch-forensischen Medizin sowie mit Beiträgen von Juristen, Kriminologen und Sozialwissenschaftlern. Entsprechend weit ist auch der Leserkreis zu fassen, der in diesem Werk Informationen finden wird. In sieben gut strukturierten und mit Zusammenfassungen versehenen Kapiteln werden auf 344 Seiten zunächst ein historischer Rückblick (reichend bis in die unmittelbare Gegenwart, Stichwort Guantanamo) sowie ethische und gesetzliche Grundlagen (z.B. zu Hungerstreik, Hafterstehungsfähigkeit, Massnahmenvollzug, Polizeigewahrsam, um nur einige zu nennen) diskutiert.
Medizinmonopol und «Bifunktionalität» der ärztlichen Rolle

Gewicht gelegt wird auf die Besonderheiten der Medizin im Strafvollzug und auf die z.T. antinomischen Aufgaben und Probleme der «therapeutischen Zwangsgemeinschaft», vor welche die im Strafvollzug Tätigen zwingend gestellt sind. Unter dem Massstab der (gesetzlich geforderten) Äquivalenz werden in einem grösseren Kapitel die Herausforderungen des «state of the art» bei Behandlung von Drogenabhängigkeit, Infektionskrankheiten, psychischen und psychiatrischen Problemen sowie Suizid und Selbstschädigung diskutiert.

Im letzten Kapitel werden schliesslich neue Ansätze zur Gesundheitsförderung (auch der betrieblichen) sowie der Qualitätsentwicklung und -verbesserung in der Institution Gefängnis besprochen. Bei zunehmender Ressourcenknappheit, bereits brisant bei der Rekrutierung von fachlich qualifiziertem Personal, werden mögliche Lösungskonzepte wie strukturelle Vernetzungen (nicht nur seitens eines im Aufbau befindlichen Informatiksystems) skizziert. Das Kapitel schliesst mit Überlegungen zur Organisation eines «Curriculum für die ärzt-liche Arbeit im Justizvollzug», Vorschläge, die sicher nicht nur für unser Nachbarland vordringlich sind.

Der Blick über die Grenze ist durch Beiträge aus der Schweiz und Österreich sichergestellt. Organisation oder Projekte der Nachbarländer werden in den jeweiligen Kapiteln optisch hervorgehoben. Das Buch wird mit einem Glossar gefängnistypischer Ausdrücke und einer umfassenden Literaturliste, geordnet nach den Buchkapiteln, ergänzt.

An dieser Stelle soll noch auf das 2006 bei Editions Médecine et Hygiène erschienene Werk «Médecine, santé et prison», herausgegeben von G. Niveau und D. Bertrand [1] mit Beiträgen zahlreicher Ärzte, vornehmlich aus der Romandie, hingewiesen werden, das als ein «Vorläufer» des hier besprochenen Buches angesehen werden kann.

Dr. med. Helena Slama, Vorstand Konferenz der Schweizerischen Gefängnisärzte 